\title{
TOPOLOGICAL ANDRÉ-QUILLEN HOMOLOGY FOR CELLULAR COMMUTATIVE $S$-ALGEBRAS
}

\author{
ANDREW BAKER, HELEN GILMOUR, AND PHILIPP REINHARD
}

\begin{abstract}
Topological André-Quillen homology for commutative $S$-algebras was introduced by Basterra following work of Kriz, and has been intensively studied by several authors. In this paper we discuss it as a homology theory on CW commutative $S$-algebras and apply it to obtain results on minimal atomic $p$-local $S$-algebras which generalise those of Baker and May for $p$-local spectra and simply connected spaces. We exhibit some new examples of minimal atomic commutative $S$-algebras.
\end{abstract}

\section{INTRODUCTION}

In this paper we give an account of some results on topological André-Quillen homology and cohomology for CW commutative $A$-algebras, where $A$ is a commutative $S$-algebra. The main goal is to develop arguments based on skeletal filtrations with a view to continuing the work begun in [11] by extending results of [2] to the case of CW commutative $S$-algebras. Some of this work originally appeared in the second author's PhD thesis [9], but we go further and use it to investigate some examples. Our main sources on topological André-Quillen (co)homology include $[3,6,13,15]$.

For definiteness, we work in the model category of commutative $S$-algebras described in [7]; following the referee's suggestions we write cofibration or fibration in place of $q$-cofibration or $q$-fibration. We use the standard notions $\rightarrow, \longmapsto$ and $\stackrel{\sim}{\rightarrow}$ to denote fibrations, cofibrations and weak equivalences, respectively. For general notions of model categories, see [10]. Given a model category $\mathscr{M}$, we will denote its homotopy category by $\bar{h} \mathscr{M}$. Thus for a commutative $S$ algebra $A, \mathscr{M}_{A}$ denotes the category of $A$-modules and $\bar{h} \mathscr{M}_{A}$ denotes its homotopy category; the latter is written $\mathscr{D}_{A}$ in [7], but we follow [3]. Where necessary, we will assume that (co)fibrant replacements are made.

We will often consider a map of commutative $S$-algebras $A \longrightarrow B$. The notations $B / A$ and $B \mid A$ are used in Basterra [3] and Quillen [17] to indicate such a pair of $S$-algebras. This notation is not ideal given the appearance of algebras over and under a given one, therefore we follow the suggestions of the referee in adopting alternatives which we hope are more suitable. In particular, we use the traditional 'pair' notation when discussing (co)homology, writing $(B, A)$ for $A \longrightarrow B$, thus following [4].

Date: 08/08/2008 Version 5

arXiv:0708.2041.

2000 Mathematics Subject Classification. Primary 55P43; Secondary 13D03, 55N35, 55P48.

Key words and phrases. S-module, $S$-algebra, cell algebra, topological André-Quillen (co)homology.

A. Baker was partially supported by a YFF Norwegian Research Council grant while at the University of Oslo in 2007-8, and Intas grants 03-51-3251 and 06-1000017-8609; H. Gilmour was supported by an EPSRC studentship; P. Reinhard was supported by an ORS grant. We would like to thank M. Basterra, P. Kropholler, M. Mandell, P. May, B. Richter, J. Rognes and S. Sagave for numerous helpful comments. We are also very grateful to the referee for encouraging us to rethink significantly issues of notation and structure, thus improving the structure of the paper. 


\section{Recollections on Topological André-Quillen (CO)homology}

Throughout, we always assume that $A$ be a cofibrant commutative $S$-algebra. We recall from [3] that for a pair of commutative $S$-algebras $(B, A)$, there is a $B$-module $\Omega_{A}(B)$ (well defind in the homotopy category $\bar{h} \mathscr{M}_{B}$ ) for which

$$
\bar{h} \mathscr{C}_{A} / B(B, B \vee M) \cong \bar{h} \mathscr{M}_{B}\left(\Omega_{A}(B), M\right) .
$$

Here $\bar{h} \mathscr{C}_{A} / B$ denotes the derived category of commutative $A$-algebras over $B$. Notice that the identity map on $\Omega_{A}(B)$ corresponds to a morphism $B \longrightarrow B \vee \Omega_{A}(B)$ which projects onto the universal derivation

$$
\delta_{(B, A)} \in \bar{h} \mathscr{M}_{A}\left(B, \Omega_{A}(B)\right) .
$$

The topological André-Quillen homology and cohomology of $(B, A)$ with coefficients in a $B$ module $M$ are defined by

$$
\begin{aligned}
& \operatorname{TAQ}_{*}(B, A ; M)=\pi_{*} \Omega_{A}(B) \wedge_{B} M, \\
& \operatorname{TAQ}^{*}(B, A ; M)=\pi_{-*} F_{B}\left(\Omega_{A}(B), M\right),
\end{aligned}
$$

where $F_{B}$ denotes the internal function object in $\mathscr{M}_{B}$.

Associated to an $A$-algebra map $B \longrightarrow C$, there are natural long exact sequences

$$
\begin{aligned}
\cdots \longrightarrow \mathrm{TAQ}_{k}(B, A ; M) \longrightarrow \mathrm{TAQ}_{k}(C, A ; M) & \longrightarrow \mathrm{TAQ}_{k}(C, B ; M) \\
& \longrightarrow \mathrm{TAQ}_{k-1}(B, A ; M) \longrightarrow \cdots \\
\cdots \longrightarrow \mathrm{TAQ}^{k}(C, B ; M) \longrightarrow \mathrm{TAQ}^{k}(C, A ; M) \longrightarrow \mathrm{TAQ}^{k}(B, A ; M) & \longrightarrow \mathrm{TAQ}^{k+1}(C, B ; M) \longrightarrow \cdots
\end{aligned}
$$

Given a pair $(B, A)$, suppose that $E$ is a $B$ ring spectrum with product $\mu: E \wedge_{B} E \longrightarrow E$, unit $j: B \longrightarrow E$ and induced unit over $A, j_{0}: A \longrightarrow B \stackrel{j}{\rightarrow} E$. Then suppressing mention of natural isomorphisms of $A$-modules of form $A \wedge_{A} X \cong X$, we obtain a commutative diagram in the homotopy category $\bar{h} \mathscr{M}_{B}$.

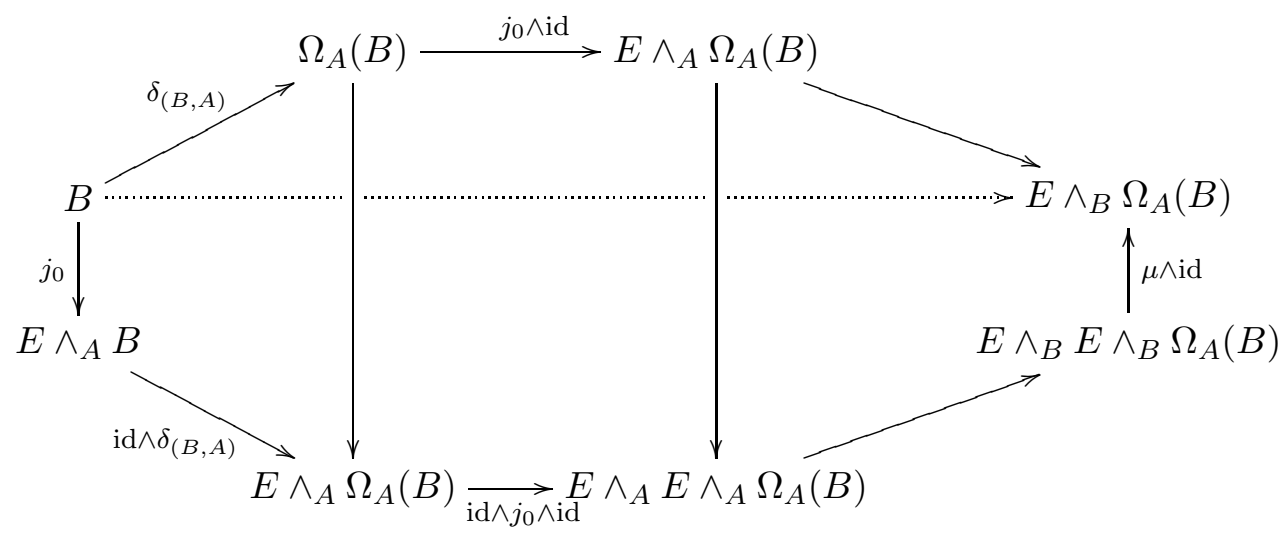

Now applying homotopy to the dotted line, i.e., the composition

$$
B \stackrel{\delta_{(B, A)}}{\longrightarrow} \Omega_{A}(B)=A \wedge_{A} \Omega_{A}(B) \longrightarrow E \wedge_{A} \Omega_{A}(B) \longrightarrow E \wedge_{B} \Omega_{A}(B),
$$

we obtain the TAQ Hurewicz homomorphism

$$
\theta: \pi_{*} B \longrightarrow \mathrm{TAQ}_{*}(B, A ; E) .
$$


Now let $\varphi: B \longrightarrow C$ be a morphism of commutative $A$-algebras for which $E$ also admits the structure of a $C$ ring spectrum so that $\varphi^{*}: \bar{h} \mathscr{M}_{C} \longrightarrow \bar{h} \mathscr{M}_{B}$ gives the same $B$ ring spectrum structure. Then the following diagram commutes, where the morphisms are the evident ones.

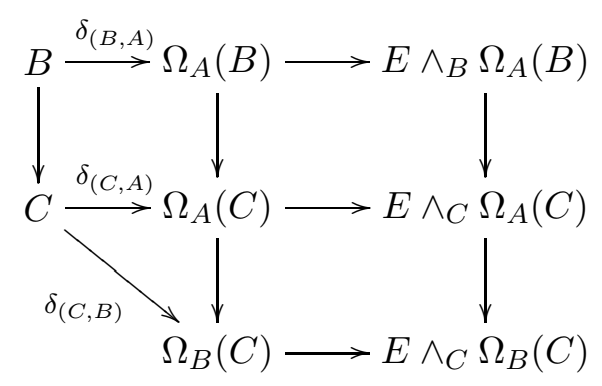

This shows that the Hurewicz homomorphism is natural with respect to morphisms of pairs $\varphi:(B, A) \longrightarrow(C, A)$ and $C$ ring spectra $E$ in the sense that the diagram

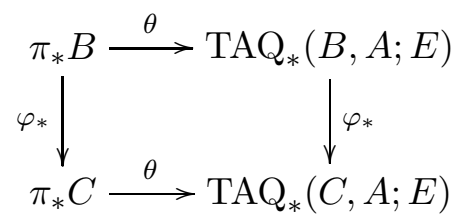

commutes. In our work we will use the Hurewicz homomorphism $\theta: \pi_{*} B \longrightarrow \mathrm{TAQ}_{*}(B, A ; E)$ when $E$ is also a commutative $B$-algebra, and use the naturality when there are morphisms of $A$-algebras $B \longrightarrow C \longrightarrow E$.

We will also use the following result which is immediate from the definition. Here we use the notation $E_{*}^{A}(-)=\pi_{*} E \wedge_{A}(-)$ and the Hurewicz homomorphism $\underline{e}: \pi_{*} B \longrightarrow E_{*}^{A} B$ is induced by the unit of $E$ over $A$.

Proposition 1.1. The TAQ Hurewicz homomorphism factors as

$$
\theta: \pi_{*} B \stackrel{e}{\rightarrow} E_{*}^{A}(B) \longrightarrow \mathrm{TAQ}_{*}(B, A ; E) .
$$

We will be especially interested in the situation where $A$ and $B$ are connective and the map $\varphi: A \longrightarrow B$ induces an isomorphism $\pi_{0} A \stackrel{\cong}{\rightarrow} \pi_{0} B$; we will write $\mathbb{k}=\pi_{0} A=\pi_{0} B$. Then there is an Eilenberg-Mac Lane object $H \mathbb{k}$, which can be taken to be a CW commutative $A$-algebra or B-algebra, which allows us to define the ordinary topological André-Quillen homology and cohomology of $(B, A)$ :

$$
\begin{aligned}
& \operatorname{HAQ}_{*}(B, A)=\operatorname{TAQ}_{*}(B, A ; H \mathbb{k})=\pi_{*} \Omega_{A}(B) \wedge_{B} H \mathbb{k}, \\
& \operatorname{HAQ}^{*}(B, A)=\operatorname{TAQ}^{*}(B, A ; H \mathbb{k})=\pi_{-*} F_{B}\left(\Omega_{A}(B), H \mathbb{k}\right) .
\end{aligned}
$$

When $\pi_{0} C=\mathbb{k}$, the long exact sequences of (1.4) yield long exact sequences in $\mathrm{HAQ}_{*}$ and $\mathrm{HAQ}^{*}$. We can also introduce coefficients in a $\mathbb{k}$-module $M$ by setting

$$
\begin{aligned}
& \operatorname{HAQ}_{*}(B, A ; M)=\operatorname{TAQ}_{*}(B, A ; H M)=\pi_{*} \Omega_{A}(B) \wedge_{B} H M, \\
& \operatorname{HAQ}^{*}(B, A ; M)=\operatorname{TAQ}^{*}(B, A ; H M)=\pi_{-*} F_{B}\left(\Omega_{A}(B), H M\right) .
\end{aligned}
$$

An important result on $\mathrm{HAQ}_{*}$ is provided by [3, lemma 8.2]. However it appears that this result is incorrectly stated (although the proof seems to be correct) and should read as follows. For a map of $A$-modules $\theta$, we denote by $\mathrm{C}_{\theta}$ the mapping cone of $\theta$ in $\mathscr{M}_{A}$.

Lemma 1.2 (Basterra [3, lemma 8.2]). Let $\varphi: A \longrightarrow B$ be an n-equivalence, where $A$ and $B$ are connective and $n \geqslant 1$. Then $\Omega_{A}(B)$ is $n$-connected and there is a map of $A$-modules $\tau: \mathrm{C}_{\varphi} \longrightarrow \Omega_{A}(B)$ for which

$$
\tau_{*}: \pi_{n+1} \mathrm{C}_{\varphi} \stackrel{\cong}{\rightarrow} \pi_{n+1} \Omega_{A}(B) .
$$


An immediate consequence is an analogue of the classical Hurewicz theorem.

Corollary 1.3. The map $\tau$ induces isomorphisms

$$
\tau_{*}: \pi_{k} \mathrm{C}_{\varphi} \cong \mathrm{HAQ}_{k}(B, A) \quad(k \leqslant n+1) .
$$

Proof. From [7] there is a Künneth spectral sequence for which

$$
\mathrm{E}_{p, q}^{2}=\operatorname{Tor}_{p, q}^{B_{*}}\left(\pi_{*}\left(\Omega_{A}(B)\right), \mathbb{k}\right) \Longrightarrow \pi_{p+q} \Omega_{A}(B) \wedge_{B} H \mathbb{k}=\mathrm{HAQ}_{p+q}(B, A)
$$

For dimensional reasons we have $\mathrm{E}_{0, n+1}^{\infty}=\mathrm{E}_{0, n+1}^{2}$ and so, on recalling that $\mathbb{k}=\pi_{0} A=\pi_{0} B$,

$$
\mathrm{HAQ}_{n+1}(B, A)=\left[\pi_{*}\left(\Omega_{A}(B)\right) \otimes_{B_{*}} \mathbb{k}\right]_{n+1}=\pi_{n+1} \Omega_{A}(B) \otimes_{B_{0}} \mathbb{k}=\pi_{n+1} \Omega_{A}(B) .
$$

Recall that for any $A$-module $X$, there is a free commutative $A$-algebra on $X$,

$$
\mathbb{P}_{A} X=\bigvee_{i \geqslant 0} X^{(i)} / \Sigma_{i}
$$

where $X^{(i)}$ is the $i$-th smash power over $A$. We remark that if $A \longrightarrow A^{\prime}$ is a morphism of commutative $S$-algebras, then from [7] we have

$$
\mathbb{P}_{A^{\prime}}\left(A^{\prime} \wedge_{A} X\right) \cong A^{\prime} \wedge_{A} \mathbb{P}_{A} X
$$

The $A$-algebra map $\mathbb{P}_{A} X \longrightarrow \mathbb{P}_{A^{*}}=A$ induced by collapsing $X$ to a point makes $A$ into an $\mathbb{P}_{A} X$-algebra, and allows us to view $\mathbb{P}_{A} X$ as an augmented $A$-algebra. There is a cofibration sequence of $\mathbb{P}_{A} X$-modules

$$
\mathbb{A}_{A} X \longrightarrow \mathbb{P}_{A} X \stackrel{\varepsilon}{\longrightarrow} \mathbb{P}_{A^{*}}=A
$$

where

$$
\mathbb{A}_{A} X=\bigvee_{i \geqslant 1} X^{(i)} / \Sigma_{i}
$$

and $\varepsilon$ is the augmentation. We note that $\mathbb{A}_{A} X$ is an $A$-nuca, i.e., a non-unital commutative $A$ algebra. For the $A$-sphere $S^{n}=S_{A}^{n}$ with $n>0$, we obtain the commutative $A$-algebra $\mathbb{P}_{A} S^{n}$ and augmentation $\mathbb{P}_{A} S^{n} \longrightarrow A$; this allows us to view an $A$-module or algebra as a $\mathbb{P}_{A} S^{n}$-module or algebra.

For a pair $(B, A)$, the $B$-module $\Omega_{A}(B)$ is defined in the homotopy category $\bar{h} \mathscr{M}_{B}$ by

$$
\Omega_{A}(B)=\mathrm{L} Q_{B} \mathrm{R} I_{B}\left(B^{c} \wedge_{A} B\right) .
$$

Here we are using the following notation in keeping with [3]. Thus $(-)^{c}$ is a cofibrant replacement functor, while $\mathrm{R} I_{B}$ is the right derived functor of the augmentation ideal functor on the category of $B$-algebras augmented over $B$. The targets of $I_{B}$ and $\mathrm{R} I_{B}$ are the category of $B$-nucas (nonunital $B$-algebras) and its homotopy category. Also, $\mathrm{L} Q_{B}$ is the left derived functor of $Q_{B}$, defined by the following strict pushout diagram in the category of $B$-modules.

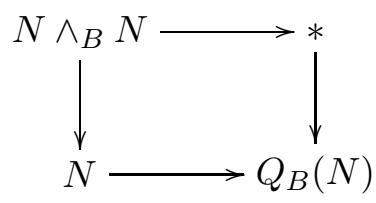

Lemma 1.4. Let $A$ be a commutative $S$-algebra, let $N$ be an $A$-nuca and let $B$ be an A-algebra. Then

$$
Q_{B}\left(B \wedge_{A} N\right)=B \wedge_{A} Q_{A}(N) .
$$


Proof. This follows from the identities

$$
\begin{gathered}
B \wedge_{A} *=* \\
B \wedge_{A} N \wedge_{B} B \wedge_{A} N \cong B \wedge_{A} N \wedge_{A} N,
\end{gathered}
$$

together with the fact that the functor $B \wedge_{A}(-)$ is a left adjoint in the category of modules, hence it respects colimits.

For an $A$-module $M$ and $A$-nuca $N$, the $A$-nuca $\mathbb{A}_{A} X$ satisfies the adjunction isomorphism

$$
\mathscr{M}_{A}(X, N) \cong \mathscr{N}_{A}\left(\mathbb{A}_{A} X, N\right),
$$

where $\mathscr{N}_{A}$ denotes the category of $A$-nucas. The idea for the proof of our next result was due to Maria Basterra.

Lemma 1.5. For each A-module $M$, there is a natural isomorphism

$$
\mathscr{M}_{A}\left(Q_{A}\left(\mathbb{A}_{A} X\right), M\right) \cong \mathscr{M}_{A}(X, M),
$$

hence $Q_{A}\left(\mathbb{A}_{A} X\right) \cong X$.

Proof. Given the natural isomorphism, Yoneda's lemma implies that $Q_{A}\left(\mathbb{A}_{A} X\right) \cong X$.

Recall Basterra's functor $Z: \mathscr{M}_{A} \longrightarrow \mathscr{N}_{A}$ which assigns to each $A$-module $M$ the same module $Z(M)$ with the trivial product $Z(M) \wedge_{A} Z(M) \longrightarrow Z(M)$. Then for any $A$-nuca $N$ we have

$$
\mathscr{M}_{A}\left(Q_{A}(N), M\right) \cong \mathscr{N}_{A}(N, Z(M)),
$$

since an $A$-module map $Q_{A}(N) \longrightarrow M$ fits into a commutative diagram

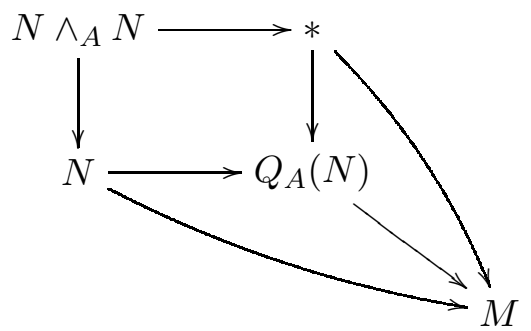

in which the rectangle is a pushout diagram. Hence there is a factorisation

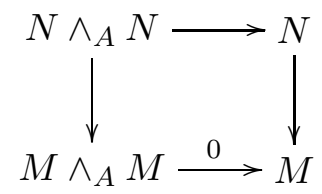

of the products, while for an $A$-nuca map $N \longrightarrow Z(M)$ there is a factorisation

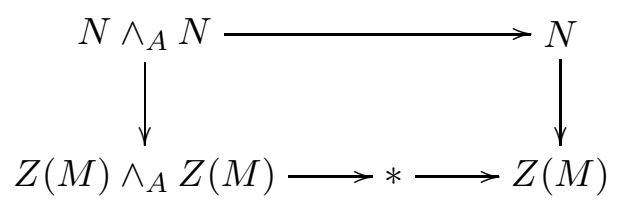

showing that the map factors through a module map $Q_{A}(N) \longrightarrow M$.

Now for any $A$-module $X$, we have

$$
\left.\mathscr{M}_{A}\left(Q_{A}\left(\mathbb{A}_{A} X\right)\right), M\right) \cong \mathscr{N}_{A}\left(\mathbb{A}_{A} X, Z(M)\right) \cong \mathscr{M}_{A}(X, M),
$$

where the second isomorphism is a consequence of the universal property of $\mathbb{A}_{A} X$. 
We will need to know the value of $\mathrm{HAQ}_{*}$ on sphere objects, and here is the basic result required for this. This was proved in the case of $A=S$ in lemma 3.6 and example 3.8 of Kuhn [14] using stabilisation, and the base change follows from the above results. For completeness, we present a more direct proof along similar lines to that given by McCarthy and Minasian [16] in the proof of theorem 6.1; unfortunately their argument appears to be incorrect as stated (at one stage they incorrectly assume that $M$ is an algebra).

Proposition 1.6. Let $X$ be a cofibrant A-module, so that $\mathbb{P}_{A} X$ is a cofibrant A-algebra. Then we have

$$
\Omega_{A}\left(\mathbb{P}_{A} X\right) \simeq \mathbb{P}_{A} X \wedge_{A} X
$$

Proof. For every $M \in \mathscr{M}_{\mathbb{P}_{A} X}$ there is an adjunction

$$
\mathscr{C}_{A} / \mathbb{P}_{A} X\left(\mathbb{P}_{A} X, \mathbb{P}_{A} X \vee M\right) \cong \mathscr{M}_{A} / \mathbb{P}_{A} X\left(X, \mathbb{P}_{A} X \vee M\right),
$$

where $\mathscr{M}_{A} / \mathbb{P}_{A} X$ denotes the category of $A$-modules over $\mathbb{P}_{A} X$. Because the forgetful functor $\mathscr{C}_{A} / \mathbb{P}_{A} X \longrightarrow \mathscr{M}_{A} / \mathbb{P}_{A} X$ respects fibrations and acyclic fibrations, the adjunction passes to homotopy categories. Now we have

$$
\mathscr{M}_{A} / \mathbb{P}_{A} X\left(\mathbb{P}_{A} X, M\right) \cong \mathscr{M}_{A} / X(X, X \vee M)
$$

and this passes to an adjunction between homotopy categories,

$$
\bar{h} \mathscr{M}_{A} / \mathbb{P}_{A} X\left(\mathbb{P}_{A} X, M\right) \cong \bar{h} \mathscr{M}_{A} / X(X, X \vee M) .
$$

Since in the homotopy category $X \vee M$ is the product of $X$ and $M$, we have

$$
\bar{h} \mathscr{M}_{A} / X(X, X \vee M) \cong \bar{h} \mathscr{M}_{A}(X, M) \text {. }
$$

By using the free functor from $A$-modules to $\mathbb{P}_{A} X$-modules, we obtain

$$
\bar{h} \mathscr{M}_{A} / X(X, X \vee M) \cong \bar{h} \mathscr{M}_{\mathbb{P}_{A} X}\left(\mathbb{P}_{A} X \wedge_{A} X, M\right) .
$$

Thus we have shown that

$$
\begin{aligned}
\bar{h} \mathscr{M}_{\mathbb{P}_{A} X}\left(\Omega_{A}\left(\mathbb{P}_{A} X\right), M\right) & \cong \bar{h} \mathscr{C}_{A} / \mathbb{P}_{A} X\left(\mathbb{P}_{A} X, \mathbb{P}_{A} X \vee M\right) \\
& \cong \bar{h} \mathscr{M}_{\mathbb{P}_{A} X}\left(\mathbb{P}_{A} X \wedge_{A} X, M\right)
\end{aligned}
$$

Now using Yoneda's lemma, we have the desired equivalence.

$$
\Omega_{A}\left(\mathbb{P}_{A} X\right) \simeq \mathbb{P}_{A} X \wedge_{A} X .
$$

On taking $B=\mathbb{P}_{A} X$ and $C=A$, the cofibration sequence of [3, proposition 4.2] yields the cofibration sequence of $A$-modules

$$
\Omega_{A}\left(\mathbb{P}_{A} X\right) \wedge_{\mathbb{P}_{A} X} A \longrightarrow \Omega_{A}(A) \longrightarrow \Omega_{\mathbb{P}_{A} X}(A)
$$

in which $\Omega_{A}(A) \simeq *$. Hence as $A$-modules,

$$
\Omega_{\mathbb{P}_{A} X}(A) \simeq \Sigma \Omega_{A}\left(\mathbb{P}_{A} X\right) \wedge_{\mathbb{P}_{A} X} A \simeq \Sigma X,
$$

where the second equivalence comes from Proposition 1.6.

Proposition 1.7. For any $\mathbb{P}_{A} S^{n}$-module $M$ we have

$$
\mathrm{TAQ}_{*}\left(\mathbb{P}_{A} S^{n}, A ; M\right) \cong M_{*-n}, \quad \mathrm{TAQ}^{*}\left(\mathbb{P}_{A} S^{n}, A ; M\right) \cong M^{*-n} .
$$

In particular,

$$
\mathrm{HAQ}_{k}\left(\mathbb{P}_{A} S^{n}, A\right)=\operatorname{HAQ}^{k}\left(\mathbb{P}_{A} S^{n}, A\right)= \begin{cases}\mathbb{k} & \text { if } k=n, \\ 0 & \text { otherwise }\end{cases}
$$


Proof. Taking $X=S^{n}$ in Proposition 1.6 we obtain

$$
\begin{aligned}
& \mathrm{TAQ}_{*}\left(\mathbb{P}_{A} S^{n}, A ; M\right)=\pi_{*} \Omega_{A}\left(\mathbb{P}_{A} S^{n}\right) \wedge_{\mathbb{P}_{A} S^{n}} M=\pi_{*} S^{n} \wedge M \cong M_{*-n}, \\
& \operatorname{TAQ}^{*}\left(\mathbb{P}_{A} S^{n}, A ; M\right)=\pi_{-*} F_{\mathbb{P}_{A} S^{n}}\left(\Omega_{A}\left(\mathbb{P}_{A} S^{n}\right), M\right)=\pi_{-*} F\left(S^{n}, M\right) \cong M^{*-n} .
\end{aligned}
$$

When $M=H \mathbb{k}$ with $\mathbb{k}=\pi_{0} A$ this gives

$$
\mathrm{HAQ}_{k}\left(\mathbb{P}_{A} S^{n}, A\right)=\operatorname{HAQ}^{k}\left(\mathbb{P}_{A} S^{n}, A\right)= \begin{cases}\mathbb{k} & \text { if } k=n, \\ 0 & \text { otherwise }\end{cases}
$$

as claimed.

Proposition 1.8. We have

$$
\operatorname{HAQ}_{k}\left(A, \mathbb{P}_{A} S^{n}\right)=\operatorname{HAQ}^{k}\left(A, \mathbb{P}_{A} S^{n}\right)= \begin{cases}\mathbb{k} & \text { if } k=n+1, \\ 0 & \text { otherwise. }\end{cases}
$$

Proof. Taking $X=S^{n}$ in (1.16) we have

$$
\begin{aligned}
\Omega_{\mathbb{P}_{A} S^{n}}(A) \wedge_{A} H \mathbb{k} & \simeq \Sigma \Omega_{A}\left(\mathbb{P}_{A} S^{n}\right) \wedge_{\mathbb{P}_{A} S^{n}} A \wedge_{A} H \mathbb{k} \simeq \Sigma S^{n} \wedge_{A} H \mathbb{k}, \\
F_{A}\left(\Omega_{\mathbb{P}_{A} S^{n}}(A), H \mathbb{k}\right) & \simeq F_{A}\left(\Sigma \Omega_{A}\left(\mathbb{P}_{A} S^{n}\right) \wedge_{\mathbb{P}_{A} S^{n}} A, H \mathbb{k}\right) \simeq F_{A}\left(\Sigma S^{n}, H \mathbb{k}\right) .
\end{aligned}
$$

Now using Proposition 1.7, the result is immediate.

We say that the $S$-algebra $A$ is simply connected if it is connective and $\pi_{0} A=\mathbb{Z}$. Our next result is an analogue of a standard result on connective spectra. Here we are considering $\operatorname{HAQ}_{*}(A, S)=\mathrm{HAQ}_{*}(A, S ; \mathbb{Z})$.

Proposition 1.9. Let $\varphi: A \longrightarrow B$ be a map of simply connected commutative $S$-algebras. Then $\varphi$ is an equivalence if and only if $\varphi_{*}: \operatorname{HAQ}_{*}(A, S) \longrightarrow \mathrm{HAQ}_{*}(B, S)$ is an isomorphism. In particular, the unit $S \longrightarrow B$ of such an $S$-algebra is a weak equivalence if and only if $\mathrm{HAQ}_{*}(B, S)=0$.

Proof. Let $n \geqslant 0$. Then $\varphi$ is an $n$-equivalence if and only if the mapping cone $\mathrm{C}_{\varphi}$ is $n$-connected. But on combining Corollary 1.3 with the long exact sequence of (1.10a), we see that $\mathrm{C}_{\varphi}$ is $n$ connected if and only if $\varphi_{*}: \mathrm{HAQ}_{k}(A, S) \longrightarrow \operatorname{HAQ}_{k}(B, S)$ is an isomorphism for all $k \leqslant n$. Since this holds for all $n$, the result follows.

As a corollary we have an analogue of the Hurewicz isomorphism theorem.

Corollary 1.10. Let $A$ be a commutative $S$-algebra whose unit $\eta: S \longrightarrow A$ is an n-equivalence. Then the Hurewicz homomorphism $\theta: \pi_{n+1} A \longrightarrow \mathrm{HAQ}_{n+1}(A, S)$ induces a monomorphism

$$
\theta^{\prime}: \pi_{n+1} A / \eta_{*} \pi_{n+1} S \longrightarrow \mathrm{HAQ}_{n+1}(A, S)
$$

which is an isomorphism if $\eta_{*}: \pi_{n} S \longrightarrow \pi_{n} A$ is a monomorphism.

Here is a slightly different way to interpret the TAQ Hurewicz homomorphism. Let $S_{A}^{n}$ denote the free $A$-module generated by the sphere $S^{n}$. For a commutative $A$-algebra $B$ there for is a Quillen adjunction

$$
\mathscr{M}_{A}\left(S_{A}^{n}, B\right) \leftrightarrows \mathscr{C}_{A}\left(\mathbb{P}_{A} S_{A}^{n}, B\right)
$$

which gives rise to an isomorphism

$$
\pi_{n} B=\bar{h} \mathscr{M}_{A}\left(S_{A}^{n}, B\right) \cong \bar{h} \mathscr{C}_{A}\left(\mathbb{P}_{A} S_{A}^{n}, B\right) .
$$

So to each homotopy class $[f] \in \pi_{n} B$ we may assign the homotopy class of $f^{\prime} \in \mathscr{C}_{A}\left(\mathbb{P}_{A} S_{A}^{n}, B\right)$. Then for any $B$ ring spectrum $E$, the induced homomorphism

$$
\Theta: A_{0} \cong \pi_{n} S_{A}^{n} \longrightarrow E_{n}^{A} S_{A}^{n} \stackrel{\cong}{\rightarrow} \mathrm{TAQ}_{n}\left(\mathbb{P}_{A} S^{n}, A ; E\right) \stackrel{f_{*}^{\prime}}{\rightarrow} \mathrm{TAQ}_{n}(B, A ; E)
$$


is related to the TAQ Hurewicz homomorphism by

$$
\theta: \pi_{n} B \longrightarrow \mathrm{TAQ}_{n}(B, A ; E), \quad \theta([f])=\Theta(1) .
$$

A particularly important case occurs when $A=S$, and $E=H \pi_{0} B$.

\section{Topological André-Quillen homology for cell $S$-algebras}

We will apply the results of Section 1 to the case of a CW commutative $S$-algebra $R$ which is the colimit of a sequence of cofibrations of cofibrant commutative $S$-algebras:

$$
S=R_{[0]} \stackrel{i_{0}}{\longrightarrow} \cdots \stackrel{i_{n-1}}{\longrightarrow} R_{[n]} \stackrel{i_{n}}{\longrightarrow} R_{[n+1]} \stackrel{i_{n+1}}{\longrightarrow} \cdots .
$$

We will also assume that only cells of degree greater than 1 are attached, thus $R_{[1]}=R_{[0]}=S$ and $\pi_{0} R=\pi_{0} S$, so these $S$-algebras are simply connected. The $(n+1)$-skeleton $R_{[n+1]}$ is obtained by attaching a wedge of $(n+1)$-cells to $R_{[n]}$ using a map $k_{n}: K_{n} \longrightarrow R_{[n]}$ from a wedge of $n$-spheres $K_{n}$ and its extension to a map of $S$-algebras $\mathbb{P}_{S} K_{n} \longrightarrow R_{[n]}$. To make this work properly, we need to use cofibrant replacement at appropriate places.

So assume as an induction hypothesis inductively that $R_{[n]}$ is defined and is a cofibrant $S$ algebra. Let $R_{[n]}^{\prime}$ be a cofibrant replacement for $R_{[n]}$ as an $\mathbb{P}_{S} K_{n}$-algebra, thus there is an acyclic fibration $R_{[n]}^{\prime} \rightarrow R_{[n]}$.

Now define $R_{[n+1]}$ and $R_{[n+1]}^{\prime \prime}$ to be the pushouts of the diagrams

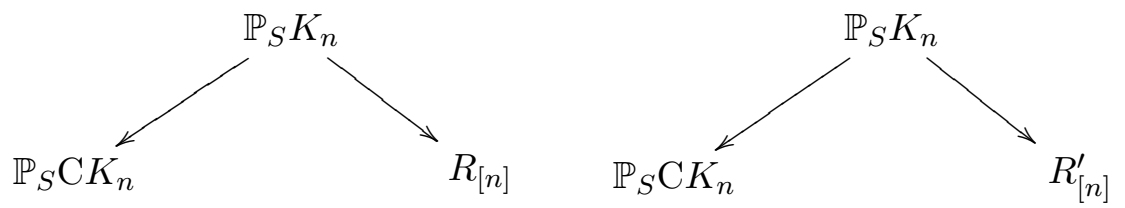

which are given by

$$
R_{[n+1]}=\mathbb{P}_{S} \mathrm{C} K_{n} \wedge_{\mathbb{P}_{S} K_{n}} R_{[n]}, \quad R_{[n+1]}^{\prime \prime}=\mathbb{P}_{S} \mathrm{C} K_{n} \wedge_{\mathbb{P}_{S} K_{n}} R_{[n]}^{\prime} .
$$

Now consider the diagram of commutative $\mathbb{P}_{S} K_{n}$-algebras (and hence of $S$-algebras)

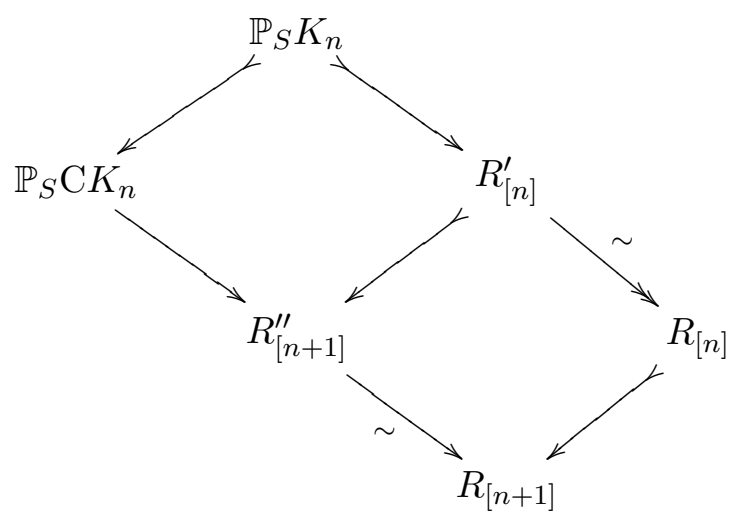

in which the upper and composite parallelograms are pushout diagrams. Since the top left hand arrow is a cofibration, the parallel ones are also cofibrations as they are pushouts. Also, by [7, proposition VII.7.4] the functor $\mathbb{P}_{S} \mathrm{C} K_{n} \wedge_{\mathbb{P}_{S} K_{n}}(-)$ preserves weak equivalences between cofibrant $S$-algebras, therefore the lower left arrow is also a weak equivalence.

Notice that for each $n$, there is a lifting diagram of the form

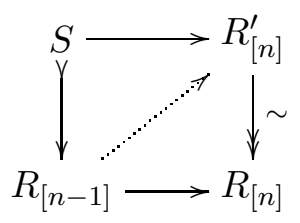


so it does no harm to assume that at each stage we have replaced $R_{[n]}$ by $R_{[n]}^{\prime}$ in what follows.

Now by [3, proposition 4.6], for a cofibrant $S$-algebra $A$ and cofibrant $A$-algebras $A \longrightarrow B$ and $A \longrightarrow C$, we have

$$
\Omega_{C}\left(B \wedge_{A} C\right) \simeq \Omega_{A}(B) \wedge_{A} C
$$

For $n \geqslant 1$ this gives

$$
\Omega_{R_{[n]}}\left(\mathbb{P}_{S} \mathrm{C} K_{n} \wedge_{\mathbb{P}_{S} K_{n}} R_{[n]}\right) \simeq \Omega_{\mathbb{P}_{S} K_{n}}\left(\mathbb{P}_{S} \mathrm{C} K_{n}\right) \wedge_{\mathbb{P}_{S} K_{n}} R_{[n]}
$$

and hence there is a long exact sequence derived from (1.4a),

$$
\begin{aligned}
\cdots \longrightarrow \mathrm{HAQ}_{k}\left(R_{[n]}, S\right) & \longrightarrow \mathrm{HAQ}_{k}\left(R_{[n+1]}, S\right) \longrightarrow \operatorname{HAQ}_{k}\left(R_{[n+1]}, R_{[n]}\right) \\
& \longrightarrow \operatorname{HAQ}_{k-1}\left(R_{[n]}, S\right) \longrightarrow \cdots
\end{aligned}
$$

which by (2.3) becomes

$$
\begin{aligned}
\cdots \longrightarrow \mathrm{HAQ}_{k}\left(R_{[n]}, S\right) \longrightarrow \mathrm{HAQ}_{k}\left(R_{[n+1]}, S\right) \longrightarrow \mathrm{HAQ}_{k}\left(\mathbb{P}_{S} \mathrm{C} K_{n}, \mathbb{P}_{S} K_{n}\right) & \\
& \longrightarrow \mathrm{HAQ}_{k-1}\left(R_{[n]}, S\right) \longrightarrow \cdots
\end{aligned}
$$

in which there is an equivalence of $\mathbb{P}_{S} K_{n}$-algebras

$$
\mathbb{P}_{S} \mathrm{C} K_{n} \simeq \mathbb{P}_{S} *=S .
$$

Hence we obtain the following long exact sequence

$$
\begin{aligned}
\quad \cdots \longrightarrow \mathrm{HAQ}_{k}\left(R_{[n]}, S\right) \longrightarrow \mathrm{HAQ}_{k}\left(R_{[n+1]}, S\right) \longrightarrow \operatorname{HAQ}_{k}\left(S, \mathbb{P}_{S} K_{n}\right) & \\
& \longrightarrow \operatorname{HAQ}_{k-1}\left(R_{[n]}, S\right) \longrightarrow \cdots
\end{aligned}
$$

Using Proposition 1.8, we can now give an estimate for the size of $\operatorname{HAQ}_{*}(R, S)$ when $R$ is a finite dimensional CW commutative $S$-algebra.

Proposition 2.1. Let $R$ be a $C W$ commutative $S$-algebra with cells only in degrees at most $n$. Then for $k>n, \operatorname{HAQ}_{k}(R, S)=0$.

Corollary 2.2. If $R$ has only finitely many cells, then

$$
\sum_{k=0}^{n} \operatorname{rank} \operatorname{HAQ}_{k}(R, S) \leqslant \text { number of cells. }
$$

In the category of $S$-modules there are three cofibration sequences that will concern us. We have the two cofibration sequences

$$
K_{n} \stackrel{k_{n}}{\longrightarrow} R_{[n]} \longrightarrow \mathrm{C}_{k_{n}}, \quad R_{[n]} \stackrel{i_{n}}{\longrightarrow} R_{[n+1]} \longrightarrow \mathrm{C}_{i_{n}} .
$$

From the proof of [3, lemma 8.2], there is a homotopy commutative diagram

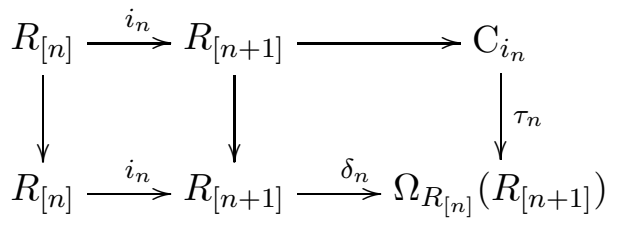


where $\delta_{n}$ denotes the universal derivation; we claim this extends to a homotopy commutative diagram of the following form.

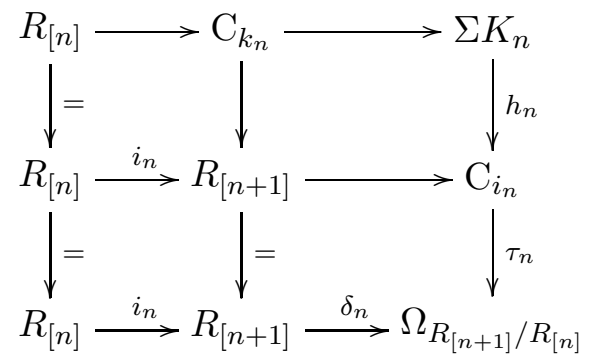

Recalling that $R_{[n+1]}$ is a pushout for the diagram of commutative $S$-algebras (2.1), the map $\mathrm{C}_{k_{n}} \longrightarrow R_{[n+1]}$ exists since $\mathrm{C}_{k_{n}}$ is defined as a pushout for the diagram of $S$-modules

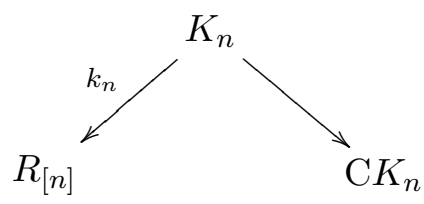

and the evident composition

$$
\mathrm{C} K_{n} \longrightarrow \mathbb{P}_{S} \mathrm{C} K_{n} \longrightarrow R_{[n+1]}
$$

gives rise to a commutative diagram of $S$-modules of the following form.

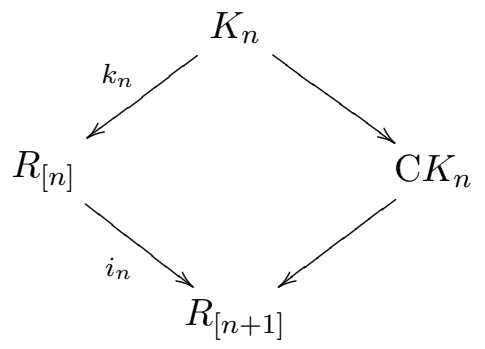

Using (2.3), we see that there are equivalences of modules over $R_{[n+1]}=R_{[n]} \wedge_{\mathbb{P}_{S} K_{n}} \mathbb{P}_{S} C K_{n}$ :

$$
\Omega_{R_{[n]}}\left(R_{[n+1]}\right) \simeq R_{[n]} \wedge_{\mathbb{P}_{S} K_{n}} \Omega_{\mathbb{P}_{S} K_{n}}(S) \simeq R_{[n]} \wedge_{\mathbb{P}_{S} K_{n}} S \wedge \Sigma K_{n} .
$$

Now smashing over $R_{[n+1]}$ with $H \mathbb{Z}$ we obtain

$$
H \mathbb{Z} \wedge_{R_{[n+1]}} \Omega_{R_{[n]}}\left(R_{[n+1]}\right) \simeq H \mathbb{Z} \wedge \Sigma K_{n} .
$$

On smashing $\tau_{n} \circ h_{n}$ with $H \mathbb{Z}$ we obtain a map

$$
H \mathbb{Z} \wedge \Sigma K_{n} \stackrel{\operatorname{id} \wedge \tau_{n} \circ h_{n}}{\longrightarrow} H \mathbb{Z} \wedge \Omega_{R_{[n]}}\left(R_{[n+1]}\right)
$$

and following this with the natural map

$$
H \mathbb{Z} \wedge \Omega_{R_{[n]}}\left(R_{[n+1]}\right) \longrightarrow H \mathbb{Z} \wedge R_{[n+1]} \Omega_{R_{[n]}}\left(R_{[n+1]}\right) \simeq H \mathbb{Z} \wedge \Sigma K_{n}
$$

yields a self map $f_{n}: H \mathbb{Z} \wedge \Sigma K_{n} \longrightarrow H \mathbb{Z} \wedge \Sigma K_{n}$. Since $K_{n}$ is a wedge of $n$-spheres, $H \mathbb{Z} \wedge K_{n}$ is a wedge of copies of $H \mathbb{Z}$. In fact, the map of (2.8) induces an isomorphism on $\pi_{n+1}(-)$.

Lemma 2.3. The map $f_{n}: H \mathbb{Z} \wedge \Sigma K_{n} \longrightarrow H \mathbb{Z} \wedge \Sigma K_{n}$ is a weak equivalence. Equivalently, the following maps are isomorphisms:

$$
\pi_{n+1} \Sigma K_{n} \stackrel{\left(h_{n}\right)_{*}}{\longrightarrow} \pi_{n+1} \mathrm{C}_{i_{n}}, \quad \pi_{n+1} \Sigma K_{n} \stackrel{\left(\tau_{n} \circ h_{n}\right)_{*}}{\longrightarrow} \pi_{n+1} \Omega_{R_{[n]}}\left(R_{[n+1]}\right) .
$$


Proof. The pairs $\left(\mathrm{C}_{k_{n}}, R_{[n]}\right)$ and $\left(R_{[n+1]}, R_{[n]}\right)$ occurring in (2.5) are relative cell complexes which have the same cells in degrees up to $2 n+1$. The cells in degree $n+1$ correspond to those on $\Sigma K_{n}$ and therefore $\left(h_{n}\right)_{*}: \pi_{n+1} \Sigma K_{n} \longrightarrow \pi_{n+1} \mathrm{C}_{i_{n}}$ is an isomorphism. For a discussion of cellular structures in this context, see [7, VII 3, X 2].

It now follows from the Hurewicz isomorphism theorem that $f_{n}$ induces an isomorphism on $\pi_{n+1}\left(H \mathbb{Z} \wedge \Sigma K_{n}\right)$ which agrees with $H_{n+1}\left(\Sigma K_{n}\right)$.

Applying homotopy to the diagram of (2.5), we obtain a diagram of abelian groups, a part of which is

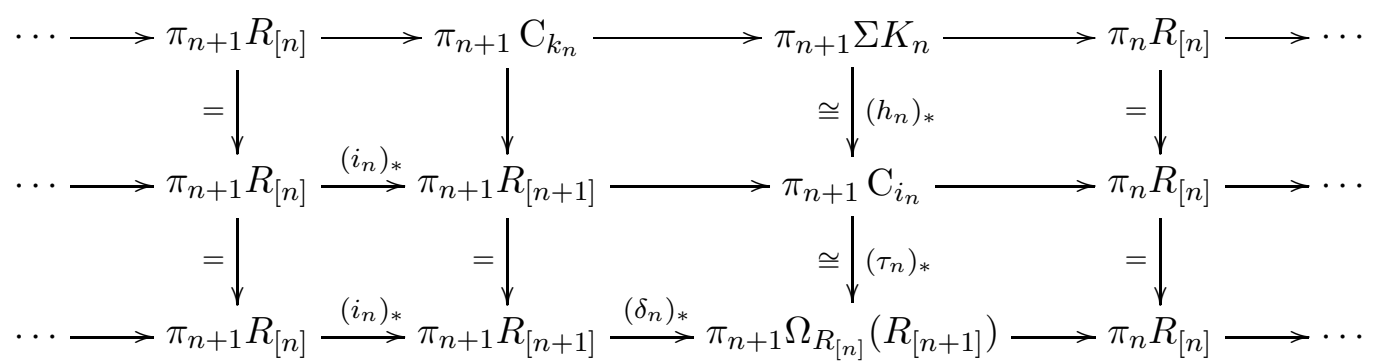

whose top two rows are exact. In the portion shown, the bottom row is also exact because $\left(\tau_{n}\right)_{*}$ is an isomorphism on $\pi_{n+1}(-)$.

Using the definition in terms of (1.6), the Hurewicz homomorphism

$$
\theta_{n+1}: \pi_{n+1} R_{[n+1]} \longrightarrow \mathrm{HAQ}_{n+1}\left(R_{[n+1]}, R_{[n]}\right)
$$

is induced from the composition

$$
R_{[n+1]} \stackrel{\delta_{\left(R_{[n+1]}, R_{[n]}\right)}}{\longrightarrow} \Omega_{R_{[n]}}\left(R_{[n+1]}\right) \longrightarrow H \mathbb{Z} \wedge_{R_{[n]}} \Omega_{R_{[n]}}\left(R_{[n+1]}\right) \longrightarrow H \mathbb{Z} \wedge_{R_{[n+1]}} \Omega_{R_{[n]}}\left(R_{[n+1]}\right),
$$

and using (1.8) it extends to a diagram

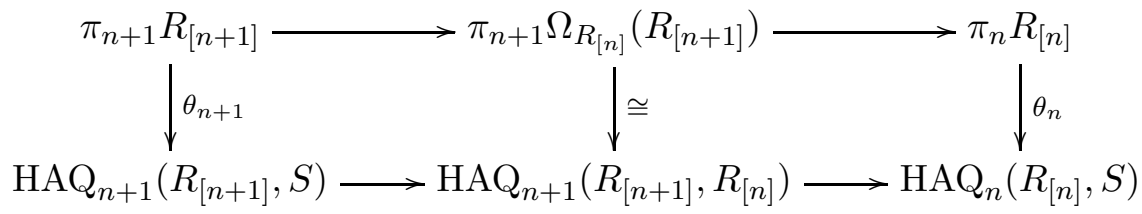

in which the bottom row is a portion of the usual long exact sequence (1.4) for $A=S, B=R_{[n]}$ and $C=R_{[n+1]}$. Furthermore, these diagrams are compatible for varying $n$.

Using the evident natural transformation $\mathrm{HAQ}_{n}(-) \longrightarrow \mathrm{HAQ}_{n}\left(-; \mathbb{F}_{p}\right)$, we can map the bottom row of (2.10) into the exact sequence

$$
0 \rightarrow \mathrm{HAQ}_{n+1}\left(R_{[n+1]}, S ; \mathbb{F}_{p}\right) \longrightarrow \mathrm{HAQ}_{n+1}\left(R_{[n+1]}, R_{[n]} ; \mathbb{F}_{p}\right) \longrightarrow \mathrm{HAQ}_{n}\left(R_{[n]}, S ; \mathbb{F}_{p}\right)
$$

to obtain the commutative diagram

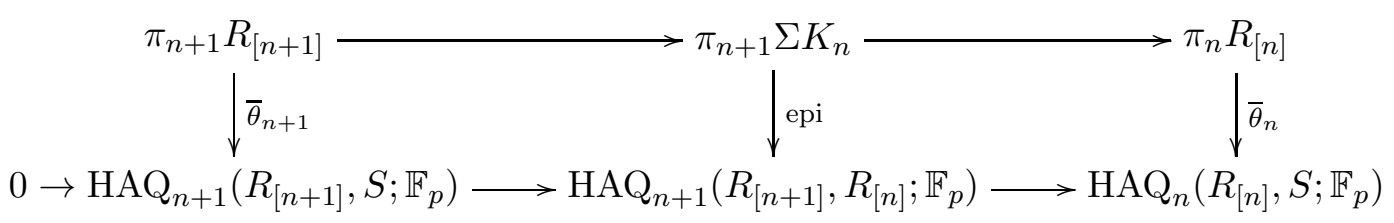

in which the rows are exact and the middle vertical arrow is an epimorphism.

Remark 2.4. All of the above works as well if we replace $S$ by the $p$-local sphere for some prime $p$, or more generally by a connective commutative $S$-algebra $A$ with $\pi_{0} A$ a localisation of $\pi_{0} S$. 


\section{Minimal atomic $p$-LOCal Commutative $S$-algebras}

From now on we fix a prime $p$. We work with $p$-local spectra, suppressing indications of localization from the notation when convenient. Thus $S$ stands for $S_{(p)}$ and so on. When discussing cell structures we always mean these to be taken $p$-locally, for example in the category of $S$-modules or $S$-algebras.

In [2], the following notion was introduced. A $p$-local CW complex $Y$ is minimal if its cellular chain complex with $\mathbb{F}_{p}$ coefficients has trivial boundaries, so

$$
\mathrm{H}_{*}\left(Y ; \mathbb{F}_{p}\right)=\mathrm{C}_{*}(Y) \otimes \mathbb{F}_{p} .
$$

An alternative formulation in terms of the skeletal inclusion maps $Y_{n} \longrightarrow Y_{n+1} \longrightarrow Y$ is that for each $n$ the induced epimorphism

$$
\mathrm{H}_{n}\left(Y_{n} ; \mathbb{F}_{p}\right) \longrightarrow \mathrm{H}_{n}\left(Y_{n+1} ; \mathbb{F}_{p}\right)
$$

is actually an isomorphism and so

$$
\mathrm{H}_{n}\left(Y_{n} ; \mathbb{F}_{p}\right) \stackrel{\cong}{\rightarrow} \mathrm{H}_{n}\left(Y_{n+1} ; \mathbb{F}_{p}\right) \stackrel{\cong}{\rightarrow} \mathrm{H}_{n}\left(Y ; \mathbb{F}_{p}\right) .
$$

In [2, theorem 3.3] it was shown that every $p$-local CW complex of finite-type is equivalent to a minimal one, so such minimal complexes exist in abundance.

Continuing to take the point of view that $\mathrm{HAQ}_{*}$ is a good substitute for ordinary homology when considering commutative $S$-algebras, let us consider the analogous notion in this multiplicative situation. We begin with a suitable definition of minimality in this context.

Definition 3.1. Let $R$ be a $p$-local CW commutative $S$-algebra with $n$-skeleton $R_{[n]}$. Then $R$ is minimal if for each $n$ the inclusion maps $R_{[n]} \longrightarrow R_{[n+1]} \longrightarrow R$ induce isomorphisms

$$
\mathrm{HAQ}_{n}\left(R_{[n]}, S ; \mathbb{F}_{p}\right) \stackrel{\cong}{\rightarrow} \mathrm{HAQ}_{n}\left(R_{[n+1]}, S ; \mathbb{F}_{p}\right)
$$

or equivalently

$$
\operatorname{HAQ}_{n}\left(R_{[n]}, S ; \mathbb{F}_{p}\right) \stackrel{\cong}{\rightarrow} \operatorname{HAQ}_{n}\left(R, S ; \mathbb{F}_{p}\right) .
$$

Proposition 2.1 implies that the homomorphisms here are both epimorphisms, as is true for their analogues in ordinary homology.

Theorem 3.2. Let $R$ be p-local $C W$ commutative $S$-algebra, with finitely many p-local cells in each degree. Then there is a minimal p-local $C W$ commutative $S$-algebra $R^{\prime}$ and an equivalence of commutative $S$-algebras $R^{\prime} \longrightarrow R$.

Proof. The details follow from the proof of [2, theorem 3.3], replacing ordinary homology with $\mathrm{HAQ}_{*}(-)$, mutatis mutandis.

This allows us to revisit the results of [2] in the context of simply connected $p$-local CW commutative $S$-algebras. The notion of a nuclear algebra appears in [11, definition 2.7], while that of an atomic algebra appears in [11, definition 2.8]. We also use notions from [2, definition 1.1]. Further details and related results appeared in the second author's PhD thesis [9].

First we have an analogue of [2, theorem 3.4]. We remark that it was not pointed out explicitly in 2] that a nuclear complex is always minimal so our next result has a direct analogue in the context of the earlier work even though it did not appear in the published version.

Theorem 3.3. Let $R$ be a simply connected p-local $C W$ commutative $S$-algebra. Then $R$ is nuclear if and only if it is minimal and the Hurewicz homomorphism $\theta: \pi_{n} R \longrightarrow \operatorname{HAQ}_{n}\left(R, S ; \mathbb{F}_{p}\right)$ is trivial for all $n>0$. 
Proof. Suppose that $R$ is nuclear in the discussion of Section 2. Then as there is a factorisation

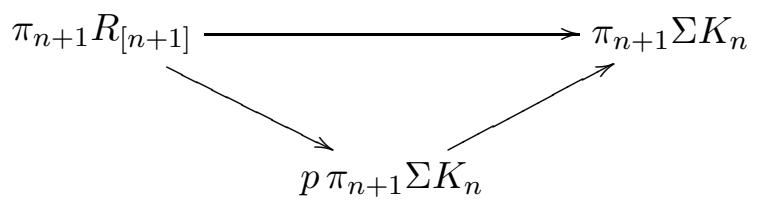

we have $\bar{\theta}_{n+1}=0$ for $n \geqslant 0$, and also

$$
\mathrm{HAQ}_{0}\left(R_{[0]}, S ; \mathbb{F}_{p}\right)=\operatorname{HAQ}_{0}\left(S, S ; \mathbb{F}_{p}\right)=0 .
$$

Similarly, the image of the boundary map $\mathrm{HAQ}_{n+1}\left(R_{[n+1]}, R_{[n]} ; \mathbb{F}_{p}\right) \longrightarrow \mathrm{HAQ}_{n}\left(R_{[n]}, S ; \mathbb{F}_{p}\right)$ is contained in $\operatorname{im} \bar{\theta}_{n}=0$, hence it is trivial. This shows that $R$ is minimal in the sense of Definition 3.1, and has no $\bmod p$ detectable homotopy in the sense that for $n>0$, the composition

$$
\bar{\theta}_{n}: \pi_{n} R_{[n]} \longrightarrow \operatorname{HAQ}_{n}\left(R_{[n]}, S ; \mathbb{F}_{p}\right) \longrightarrow \operatorname{HAQ}_{n}\left(R, S ; \mathbb{F}_{p}\right)
$$

is trivial.

By a similar argument to the proof of [2, theorem 3.4], the converse also holds, i.e., if $R$ is minimal and has no mod $p$ detectable homotopy then it is nuclear.

We claim that [11, conjecture 2.9] and the analogue of [2, proposition 2.5] are consequences of our next result.

Theorem 3.4. A nuclear simply connected p-local $C W$ commutative $S$-algebra is minimal atomic, hence a core of such an algebra is an equivalence.

Proof. This follows the analogous proof of [2].

We also note the following useful result.

Proposition 3.5. Let $R$ be minimal atomic as an $S$-module. Then it is minimal atomic as an S-algebra.

Proof. This follows easily from the fact that for $S$-modules, being minimal atomic is equivalent to being irreducible.

As an alternative, notice that if $R$ is minimal atomic as an $S$-module, then the ordinary homology Hurewicz homomorphism $\pi_{n} R \longrightarrow H_{n}\left(R ; \mathbb{F}_{p}\right)$ is trivial for $n>0$, so by Proposition 1.1 the mod $p$ HAQ Hurewicz homomorphism $\pi_{n} R \longrightarrow \operatorname{HAQ}_{n}\left(R, S ; \mathbb{F}_{p}\right)$ is trivial, whence $R$ is a minimal atomic $S$-algebra.

\section{The TAQ Hurewicz homomorphism for Thom spectra}

In order to calculate with Thom spectra arising from infinite loop maps into $B S F$, we need some information on the relevant universal derivations. The next two results are implicit in the proof of [4, theorem 6.1], but unfortunately they are not stated explicitly and we are grateful to Mike Mandell and Maria Basterra for clarifying this material which is due to them.

Let $X$ be an infinite loop space and let $\underline{X}$ be the associated spectrum viewed as an $S$-module. Following [4, section 6], consider the augmented $S$-algebra

$$
\Sigma_{S+}^{\infty} X=S \wedge_{\mathcal{L}} \Sigma^{\infty} X_{+} .
$$

There is a canonical (evaluation) morphism of $S$-modules $\sigma: \Sigma_{S+}^{\infty} X \longrightarrow \underline{X}$. Now taking $A=$ $B=S$ and using the isomorphism $\Sigma_{S+}^{\infty} X \wedge_{S} S \cong \Sigma_{S+}^{\infty} X$, the bijection of [3, proposition 3.2] gives

$$
\bar{h} \mathscr{C}_{S} / S\left(\Sigma_{S+}^{\infty} X, S \vee M\right) \cong \bar{h} \mathscr{M}_{S}\left(\mathrm{~L} Q_{S} \mathrm{R} I_{S}\left(\Sigma_{S+}^{\infty} X\right), M\right)
$$


When $M=\mathrm{L} Q_{S} \mathrm{R} I_{S}\left(\Sigma_{S+}^{\infty} X\right)$, the identity morphism on the right hand side corresponds to a morphism

$$
\Sigma_{S+}^{\infty} X \longrightarrow S \vee \mathrm{L} Q_{S} \mathrm{R} I_{S}\left(\Sigma_{S+}^{\infty} X\right)
$$

which projects to a universal derivation

$$
\delta_{X}: \Sigma_{S+}^{\infty} X \longrightarrow \mathrm{L} Q_{S} \mathrm{R} I_{S}\left(\Sigma_{S+}^{\infty} X\right) .
$$

Proposition 4.1. In the homotopy category $\bar{h} \mathscr{M}_{S}$ there is an isomorphism

$$
\theta: \underline{X} \stackrel{\cong}{\rightrightarrows} Q_{S} \mathrm{R} I_{S}\left(\Sigma_{S+}^{\infty} X\right)
$$

and a commutative diagram

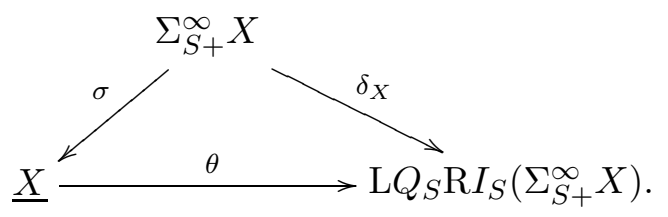

Hence $\sigma$ realises the universal derivation.

Note that the diagonal on $X$ induces a morphism of commutative $\Sigma_{S+}^{\infty} X$-algebras

$$
\Sigma_{S+}^{\infty} X \wedge_{S} \Sigma_{S+}^{\infty} X \stackrel{\text { id^diag }}{\longrightarrow} \Sigma_{S+}^{\infty} X \wedge_{S} \Sigma_{S+}^{\infty} X \wedge_{S} \Sigma_{S+}^{\infty} X \stackrel{\text { mult } \wedge \text { id }}{\longrightarrow} \Sigma_{S+}^{\infty} X \wedge_{S} \Sigma_{S+}^{\infty} X
$$

which is a weak equivalence. This has the effect of interchanging the multiplication map with the augmentation onto the second factor, giving rise to a composition of isomorphisms in $\bar{h} \mathscr{M}_{\Sigma_{S+}^{\infty} X}$, namely

$$
\Omega_{S}\left(\Sigma_{S+}^{\infty} X\right) \stackrel{\cong}{\rightrightarrows} \Sigma_{S+}^{\infty} X \wedge_{S} \mathrm{~L} Q_{S} \mathrm{R} I_{S}\left(\Sigma_{S+}^{\infty} X\right) \stackrel{\cong}{\rightrightarrows} \Sigma_{S+}^{\infty} X \wedge_{S} \underline{X} .
$$

For an infinite loop map $f: X \longrightarrow B F$, the resulting Thom spectrum $M f$ (viewed as a commutative $S$-algebra) has a Thom diagonal $\Delta: M f \longrightarrow M f \wedge_{S} \Sigma_{S+}^{\infty} X$, so that the composition

$$
M f \wedge_{S} M f \stackrel{\text { id } \wedge \Delta}{\longrightarrow} M f \wedge_{S} M f \wedge_{S} \Sigma_{S+}^{\infty} X \stackrel{\text { multıid }}{\longrightarrow} M f \wedge_{S} \Sigma_{S+}^{\infty} X
$$

is a weak equivalence of $M f$-algebras. Furthermore, the map $\sigma$ induces a map of $S$-algebras

$$
\delta_{f}: M f \stackrel{\Delta}{\rightarrow} M f \wedge_{S} \Sigma_{S+}^{\infty} X \stackrel{\operatorname{id} \wedge \sigma}{\longrightarrow} M f \wedge_{S} \underline{X}
$$

and then we have the following result in which we use the universal derivation $\delta_{(M f, S)}$ of (1.2).

Proposition 4.2. In the homotopy category $\bar{h} \mathscr{M}_{M f}$ there is an isomorphism

$$
\Theta: M f \wedge_{S} \underline{X} \stackrel{\cong}{\longrightarrow} \Omega_{S}(M f)
$$

and a commutative diagram

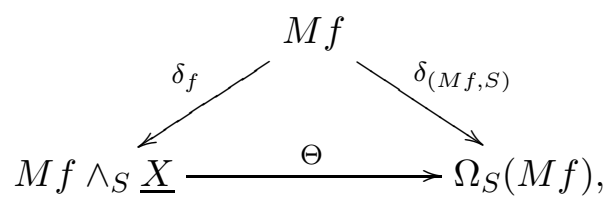

and hence $\delta_{f}$ realises the universal derivation.

Given this result, we can now describe how to calculate the HAQ Hurewicz homomorphism for a Thom spectrum $M f$ induced by an infinite loop map $f: X \longrightarrow B S F$. Recalling the definition using the map of (1.6), we can take TAQ with coefficient spectrum the integral Eilenberg-Mac Lane spectrum $H=H \mathbb{Z}$ (or indeed the Eilenberg-Mac Lane spectrum of any commutative ring) and so obtain homomorphisms

$$
\theta: \pi_{n} M f \longrightarrow \mathrm{HAQ}_{n}(M f, S)=\mathrm{TAQ}_{n}(M f, S ; H) .
$$


Then the map of (1.6) factors as in the commutative diagram obtained from (1.5),

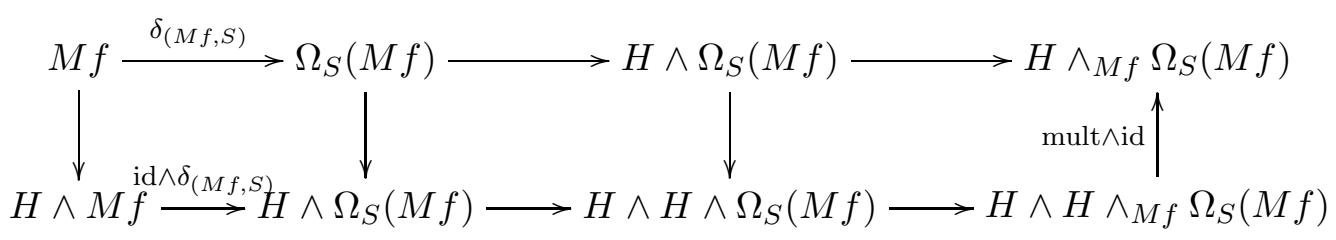

in which the undecorated smash products are taken over $S$ and the downward pointing arrows are obtained by smashing with the unit $S \longrightarrow H$. Thus $\theta$ factors through the usual Hurewicz homomorphism:

$$
\theta: \pi_{n} M f \longrightarrow H_{n}(M f) \longrightarrow \mathrm{HAQ}_{n}(M f, S) .
$$

Using Proposition 4.2 we see that $\theta$ is equivalent to the homomorphism

$$
\theta^{\prime}: \pi_{n} M f \longrightarrow H_{n}(\underline{X})=\pi_{n}(H \wedge \underline{X})
$$

induced by

$$
M f \stackrel{\Delta}{\rightarrow} M f \wedge \Sigma^{\infty} X_{+} \stackrel{U \wedge \mathrm{id}}{\longrightarrow} H \wedge \Sigma^{\infty} X_{+} \stackrel{\mathrm{id} \wedge \sigma}{\longrightarrow} H \wedge \underline{X},
$$

where $U \in H^{0}(M f ; R)$ is the orientation class. On smashing with $H$, we obtain

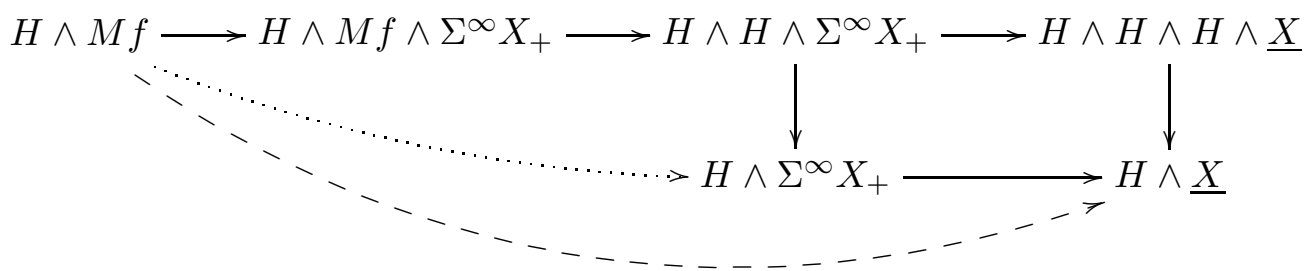

where the dashed arrow induces $\theta^{\prime \prime}: H_{n}(M f) \longrightarrow \mathrm{HAQ}_{n}(M f, S)$ and the dotted arrow induces the Thom isomorphism $H_{n}(M f) \longrightarrow H_{n}(X)$. Thus $\theta^{\prime \prime}$ is the Thom isomorphism composed with $\sigma_{*}: H_{n}(X) \longrightarrow H_{n}(\underline{X})$.

Notice that $\sigma_{*}$ factors through the homology suspension

$$
H_{n}(X) \stackrel{\cong}{\rightrightarrows} H_{n+1}(\Sigma X) \longrightarrow H_{n+1}(B X),
$$

where $B X$ is the delooping of $X$. Hence $\sigma_{*}$, and therefore $\theta^{\prime \prime}$, annihilates decomposables in the rings on which they are defined.

\section{Some examples of Thom SPeCtra}

The Thom spectrum $M U$ : By Proposition 4.2, we know that $\Omega_{S}(M U)=M U \wedge \Sigma^{2} k u$ since $B U$ is the zeroth space in the 1-connected cover of $k u$ which is $\Sigma^{2} k u$. Thus for any commutative ring $\mathbb{F}$ we have

$$
\operatorname{HAQ}_{*}(M U, S ; \mathbb{F})=H_{*}\left(\Sigma^{2} k u ; \mathbb{F}\right)=H_{*-2}(k u ; \mathbb{F}) .
$$

For the prime 2,

$$
\operatorname{HAQ}_{*}\left(M U, S ; \mathbb{F}_{2}\right)=H_{*-2}\left(k u ; \mathbb{F}_{2}\right),
$$

where the right hand side is given by

$$
H_{*}\left(k u ; \mathbb{F}_{2}\right)=\mathbb{F}_{2}\left[\zeta_{1}^{4}, \zeta_{2}^{2}, \zeta_{3}, \zeta_{4}, \ldots\right] \subseteq \mathcal{A}(2)_{*}=\mathbb{F}_{2}\left[\zeta_{1}, \zeta_{2}, \zeta_{3}, \zeta_{4}, \ldots\right],
$$

and is a sub Hopf algebra of the mod 2 dual Steenrod algebra with the inclusion induced from the natural map $k u \longrightarrow H \mathbb{F}_{2}$. The generators $\zeta_{r}$ are the conjugates of the generators $\xi_{r}$ coming from $H_{*}\left(B \mathbb{Z} / 2 ; \mathbb{F}_{2}\right)$. 
For an odd prime $p$, there is decomposition

$$
k u_{(p)} \simeq \bigvee_{0 \leqslant r \leqslant p-2} \Sigma^{2 r} \ell,
$$

where $\ell$ is the Adams summand for which

$$
H_{*}\left(\ell ; \mathbb{F}_{p}\right)=\mathbb{F}_{p}\left[\zeta_{1}, \zeta_{2}, \zeta_{3}, \ldots\right] \otimes \Lambda\left(\bar{\tau}_{2}, \bar{\tau}_{3}, \ldots\right) \subseteq \mathcal{A}(p)_{*}=\mathbb{F}_{p}\left[\zeta_{1}, \zeta_{2}, \zeta_{3}, \ldots\right] \otimes \Lambda\left(\bar{\tau}_{0}, \bar{\tau}_{1}, \ldots\right),
$$

where the embedding into the dual mod $p$ dual Steenrod algebra is induced by a map $\ell \longrightarrow$ $H \mathbb{F}_{p}$. The generators $\zeta_{r}$ and $\bar{\tau}_{r}$ are the conjugates of the generators $\xi_{r}$ and $\tau_{r}$ coming from $H_{*}\left(B \mathbb{Z} / p ; \mathbb{F}_{p}\right)$.

Recall that for a prime $p$, the image of the mod $p$ Hurewicz homomorphism $h: \pi_{*} M U \longrightarrow$ $H_{*}\left(M U ; \mathbb{F}_{p}\right)$ is

$$
h \pi_{*} M U=\mathbb{F}_{p}\left[x_{r}: r+1 \text { is not a power of } p\right] \subseteq H_{*}\left(M U ; \mathbb{F}_{p}\right),
$$

where, in the notation of Adams [1],

$$
x_{r} \equiv b_{r} \quad \text { (mod decomposables). }
$$

Of course, $x_{r}$ is the image of an element of $\pi_{2 r} M U_{(p)}$. The Thom isomorphism is

$$
\Phi: H_{*}\left(M U ; \mathbb{F}_{p}\right) \stackrel{\cong}{\rightrightarrows} H_{*}\left(B U ; \mathbb{F}_{p}\right) ; \quad b_{n} \longleftrightarrow \beta_{n},
$$

so to determine the Hurewicz homomorphism $\theta: \pi_{*} M U \longrightarrow \operatorname{HAQ}_{*}\left(M U, S ; \mathbb{F}_{p}\right)$ we need to calculate the image of $\sigma_{*}: H_{*}\left(B U ; \mathbb{F}_{p}\right) \longrightarrow H_{*-2}\left(k u ; \mathbb{F}_{p}\right)$. Since decomposables are killed by $\sigma_{*}$, it suffices to know its effect on the generators $\beta_{n}$.

When $p=2$, recall that the map $k u \longrightarrow H \mathbb{F}_{2}$ induces a monomorphism in $H_{*}\left(-; \mathbb{F}_{2}\right)$ and the canonical maps give a composition

$$
\Sigma^{\infty} \mathbb{C P}^{\infty} \longrightarrow \Sigma^{\infty} B U \longrightarrow \Sigma^{2} k u \longrightarrow \Sigma^{2} H \mathbb{F}_{2}
$$

which corresponds to the natural cohomology generator $x \in H^{2}\left(\mathbb{C P}^{\infty} ; \mathbb{F}_{2}\right)$ and the homology generator $\beta_{n}$ dual to $x^{n}$ (which maps to $\beta_{n} \in H_{2 n}\left(B U ; \mathbb{F}_{p}\right)$ ) maps to the coefficient of $t^{n}$ in the power series

$$
\xi(t)=\sum_{s \geqslant 0} \xi_{s}^{2} t^{2^{s}}
$$

This shows that

$$
\sigma_{*}\left(\beta_{n}\right) \begin{cases}\neq 0 & \text { if } n \text { is a power of } 2 \\ =0 & \text { otherwise }\end{cases}
$$

Hence we see that

$$
\theta \pi_{2 n} M U \begin{cases}\neq 0 & \text { if } n \text { is a power of } 2 \\ =0 & \text { otherwise. }\end{cases}
$$

More precisely, this shows that the polynomial generator of $\pi_{*} M U$ in degree $2 n$ is detected in $\mathrm{HAQ}_{2 n}\left(M U, S ; \mathbb{F}_{2}\right)$ if and only if $n$ is a power of 2 . Therefore $M U_{(2)}$ is not minimal atomic.

For an odd prime $p$, there is splitting of infinite loop spaces

$$
B U_{(p)} \simeq W_{1} \times \cdots \times W_{p-1},
$$

where $W_{r}$ is the zeroth space of $\Sigma^{2 r} \ell$, so that the natural map

$$
\Sigma^{\infty} B U_{(p)} \longrightarrow \Sigma^{2} k u_{(p)} \simeq \bigvee_{1 \leqslant r \leqslant p-1} \Sigma^{2 r} \ell
$$

factors through a wedge of maps $\Sigma^{\infty} W_{r} \longrightarrow \Sigma^{2 r} \ell$. An exercise in using the definition of the Adams splitting together with the fact that the lowest degree cohomology class for $W_{r}$ 
corresponds to the Newton polynomial in the Chern classes in $H^{2 r}\left(B U ; \mathbb{F}_{p}\right)$, shows that the induced composition

$$
\Sigma^{\infty} \mathbb{C P}^{\infty} \stackrel{x^{r}}{\longrightarrow} \Sigma^{\infty} W_{r} \longrightarrow \Sigma^{2 r} H \mathbb{F}_{p}
$$

has the effect on homology of sending $\beta_{n}$ to the coefficient of $t^{n}$ in the series

$$
\xi(t)^{r}=\left(\sum_{s \geqslant 0} \xi_{s} t^{p^{s}}\right)^{r}
$$

In particular, this shows that when $1 \leqslant r \leqslant p-1$, each generator $b_{p^{s}-1+r} \in H_{2\left(p^{s}-1+r\right)}\left(M U ; \mathbb{F}_{p}\right)$ survives to give a non-zero element in $H_{2\left(p^{s}-1+r\right)}\left(\Sigma^{2} k u ; \mathbb{F}_{p}\right)$, therefore the corresponding generator $x_{p^{s}-1+r} \in \pi_{2\left(p^{s}-1+r\right)} M U$ also survives to a non-zero element of $H_{2\left(p^{s}-1+r\right)}\left(\Sigma^{2} k u ; \mathbb{F}_{p}\right)$.

To summarise, the Hurewicz homomorphism $\theta: \pi_{*} M U \longrightarrow \mathrm{HAQ}_{*}\left(M U, S ; \mathbb{F}_{p}\right)$ detects homotopy, so $M U_{(p)}$ is not minimal atomic for any prime $p$.

A core for $M U_{(2)}$ : The problem of identifying a core for $M U_{(p)}$ was first studied in [1] where the following example for the case $p=2$ appeared. We give a new verification that the domain is indeed minimal atomic. Of course, it would be very interesting to identify cores of $M U$ for the odd primes.

Consider the infinite loop map $B U \longrightarrow B S p$ classifying quaternionification of complex bundles. The fibre is also an infinite loop map $j: S p / U \longrightarrow B U$, where $S p / U$ is the zeroth space of $\Sigma^{2} k o$. Then $j$ induces a Thom spectrum $M j$ which is a commutative $S$-algebra and the natural map $M j \longrightarrow M U$ is a morphism of commutative $S$-algebras. Furthermore, the associated map in 2-local homology gives an isomorphism onto half of $H_{*}\left(M U ; \mathbb{Z}_{(2)}\right)$ :

$$
H_{*}\left(M j ; \mathbb{Z}_{(2)}\right)=\mathbb{Z}_{(2)}\left[y_{2 r-1}: r \geqslant 1\right] \stackrel{\cong}{\rightarrow} \mathbb{Z}_{(2)}\left[y_{2 r-1}^{\prime}: r \geqslant 1\right] \subseteq H_{*}\left(M U ; \mathbb{Z}_{(2)}\right),
$$

where $y_{2 r-1}, y_{2 r-1}^{\prime}$ have degree $2 r-2$ and in $H_{*}\left(M U ; \mathbb{Z}_{(2)}\right)$,

$$
y_{2 r-1}^{\prime} \equiv b_{2 r-1} \quad(\bmod \text { decomposables }) .
$$

There is a similar result for mod 2 homology.

From now on in this example, all spectra are assumed to be localised at 2 and we drop this from the notation.

The argument of [1] shows that $M j$ is a wedge of suspensions of $B P$ and as the induced map in homology is a monomorphism so is that in homotopy. So to show that $M j \longrightarrow M U$ is a core we only need to show that $M j$ is minimal atomic.

This time we need to examine the mod 2 HAQ Hurewicz homomorphism which amounts to a homomorphism

$$
\pi_{*} M j \longrightarrow H_{*}\left(\Sigma^{2} k o ; \mathbb{F}_{2}\right) .
$$

By work of Stong [19, the map induced by the bottom cohomology class gives a homology monomorphism $H_{*}\left(\Sigma^{2} k o ; \mathbb{F}_{2}\right) \longrightarrow \mathcal{A}(2)_{*}$. There is a commutative diagram

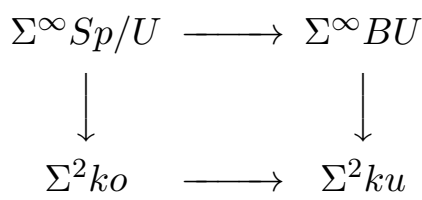

and on applying $H_{*}\left(-; \mathbb{F}_{2}\right)$ and using the calculations described for $M U$, we see that

$$
\sigma_{*}\left(y_{2 r-1}^{\prime}\right)=0 \quad(r \geqslant 1) .
$$

Hence the mod 2 HAQ Hurewicz homomorphism is also trivial in positive degrees. 
We remark that the morphisms of $S$-algebras $S \longrightarrow M j \longrightarrow M U$ give rise to a cofibre sequence of $M U$-modules

$$
M U \wedge \Sigma^{2} k o \longrightarrow M U \wedge \Sigma^{2} k u \longrightarrow \Omega_{M U / M j}
$$

and this is equivalent to the cofibre sequence

$$
M U \wedge \Sigma^{2} k o \longrightarrow M U \wedge \Sigma^{2} k u \longrightarrow M U \wedge \Sigma^{4} k o,
$$

where we view $k u$ as $k o \wedge \mathrm{C}_{\eta}$ for $\eta \in \pi_{1} S$ the generator. See [18, proposition 5.3.1] for more on this sequence in a Galois theoretic context. Smashing with $H \mathbb{F}_{2}$ over $M U$ now gives the usual short exact sequence

$$
0 \rightarrow H_{*}\left(k o ; \mathbb{F}_{2}\right) \longrightarrow H_{*}\left(k u ; \mathbb{F}_{2}\right) \longrightarrow H_{*-2}\left(k o ; \mathbb{F}_{2}\right) \rightarrow 0 .
$$

$M S p$ 2-locally: Here only the prime $p=2$ presents an interesting question.

Proposition 5.1. $M S p_{(2)}$ is minimal atomic as a commutative $S$-algebra but not as an $S$ module.

Proof. We will show that the mod 2 HAQ Hurewicz homomorphism is trivial in positive degrees. It is known that the mod 2 Hurewicz homomorphism $\pi_{*} M S p \longrightarrow H_{*}\left(M S p ; \mathbb{F}_{2}\right)$ is not trivial in positive degrees, so $M S p$ is not minimal atomic as a 2-local spectrum.

For this example, $B S p$ is the zeroth space of the 3 -connected spectrum $\Sigma^{4} k o$. From [19], the bottom integral cohomology class induces a monomorphism

$$
H_{*}\left(k s p ; \mathbb{F}_{2}\right) \longrightarrow H_{*}\left(\Sigma^{4} H \mathbb{F}_{2} ; \mathbb{F}_{2}\right)=\mathcal{A}(2)_{*-4} .
$$

However, the crux of our argument involves a beautiful result of Floyd [8] and we describe this in some detail.

Recall that the natural map $M S p \longrightarrow M O$ induces a mod 2 homology isomorphism onto the 4-th powers:

$$
H_{*}\left(M S p ; \mathbb{F}_{2}\right) \stackrel{\cong}{\rightarrow} H_{*}\left(M O ; \mathbb{F}_{2}\right)^{(4)} \subseteq H_{*}\left(M O ; \mathbb{F}_{2}\right) .
$$

In [8, theorems 5.3,5.5], it is shown that $\pi_{*} M O$ has a family of polynomial generators $z_{r} \in \pi_{r} M O$ $(r+1$ not a power of 2$)$ for which the polynomial subring

$$
P_{*}=\mathbb{F}_{2}\left[z_{r}^{\kappa(r)}: r+1 \text { not a power of } 2\right] \subseteq \pi_{*} M O,
$$

satisfies

$$
\operatorname{im}\left(\pi_{*} M S p \longrightarrow \pi_{*} M O\right) \subseteq P_{*}^{(8)},
$$

where

$$
\kappa(r)= \begin{cases}2 & \text { if } r \text { is odd or a power of } 2 \\ 1 & \text { if } r \text { is even and not a power of } 2 .\end{cases}
$$

Kochman [12] shows that these two rings are actually equal, but for our purposes it suffices to have the inclusion.

For our purposes we need only remark that (5.3) together with the fact that the Hurewicz homomorphism $\pi_{*} M O \longrightarrow H_{*}\left(M O ; \mathbb{F}_{2}\right)$ is monic, combine to show that the Hurewicz homomorphism for $M S p$ has image contained in the squares:

$$
\operatorname{im}\left(\pi_{*} M S p \longrightarrow H_{*}\left(M S p ; \mathbb{F}_{2}\right)\right) \subseteq H_{*}\left(M S p ; \mathbb{F}_{2}\right)^{(2)},
$$

hence in positive degree this image is contained in the decomposables. Therefore the composition

$$
H_{*}\left(M S p ; \mathbb{F}_{2}\right) \stackrel{\cong}{\rightarrow} H_{*}\left(B S p ; \mathbb{F}_{2}\right) \stackrel{\sigma_{*}}{\longrightarrow} H_{*}\left(\Sigma^{4} k s p ; \mathbb{F}_{2}\right)
$$

is trivial in positive degrees since the second map annihilates decomposables. 


\section{CONCLUDING REMARKS}

An important goal for this paper was to show how TAQ could be used to introduce cellular arguments into the study of commutative $S$-algebras, and in particular to aid in identifying the minimal atomic $p$-local $S$-algebras introduced in [11] using ideas suggested by [2]. We have simplified things by confining attention to simply connected examples, however the theory can be adapted to work as well when $\pi_{0} R$ is finite cyclic of prime power order, and we have already investigated some examples. It would also be interesting to have an analogous theory for cellular $S$-algebras in Bousfield localised situations, for example for $K(n)$-local algebras. We expect to address these issues in future work.

\section{REFERENCES}

[1] J. F. Adams, Stable homotopy and generalised homology, Chicago Lectures in Mathematics, University of Chicago Press, Chicago, 1974.

[2] A. J. Baker and J. P. May, Minimal atomic complexes, Topology 43 (2004), no. 2, 645-665.

[3] M. Basterra, André-Quillen cohomology of commutative S-algebras, J. Pure Appl. Algebra 144 (1999), no. 2, $111-143$.

[4] M. Basterra and M. Mandell, Homology and cohomology of $E_{\infty}$ ring spectra, Math. Z. 249 (2005), no. 4, 903-944.

[5] M. Basterra and R. McCarthy, Г-homology, topological André-Quillen homology and stabilization, Topology Appl. 121 (2002), no. 3, 551-566.

[6] M. Basterra and B. Richter, (Co-)homology theories for commutative S-algebras, Mathematical Society Lecture Note Series, Structured Ring Spectra, (2004), pp. 115-130.

[7] A. D. Elmendorf, I. Kriz, M. A. Mandell, and J. P. May, Rings, modules, and algebras in stable homotopy theory, Mathematical Surveys and Monographs, vol. 47, American Mathematical Society, 1997. with an appendix by M. Cole.

[8] E. E. Floyd, Stiefel-Whitney numbers of quaternionic and related manifolds, Trans. Am. Math. Soc. 155 (1971), no. 2, 77-94.

[9] H. Gilmour, Nuclear and minimal atomic S-algebras, PhD thesis, University of Glasgow, 2006.

[10] M. Hovey, Model categories, Mathematical Surveys and Monographs, vol. 63, American Mathematical Society, Providence, RI, 1999.

[11] P. Hu, I. Kriz, and J. P. May, Cores of spaces, spectra and $E_{\infty}$ ring spectra, Homology, Homotopy and Applications 3 (2001), no. 2, 341-54.

[12] S. O. Kochman, The symplectic cobordism ring, I, Mem. Am. Math. Soc., vol. 228, American Mathematical Society, 1980.

[13] I. Kriz, Towers of $E_{\infty}$ ring spectra with an application to BP, unpublished preprint.

[14] N. J. Kuhn, Localization of André-Quillen-Goodwillie towers, and the periodic homology of infinite loopspaces, Adv. Math. 201 (2006), no. 2, 318-378.

[15] M. A. Mandell, Topological André-Quillen cohomology and $E_{\infty}$ André-Quillen cohomology, Adv. Math. 177 (2003), no. 2, 227-279.

[16] R. McCarthy and V. Minasian, HKR theorem for smooth S-algebras, J. Pure Appl. Algebra 185 (2003), 239-258.

[17] D. Quillen, On the (co)-homology of commutative rings, Appl. Categorical Algebra, Proc. Sympos. Pure Math., 1970, pp. 65-87.

[18] J. Rognes, Galois extensions of structured ring spectra, Mem. Am. Math. Soc., vol. 192, 2008 pp. 1-97.

[19] R. E. Stong, Notes on cobordism theory, Mathematical Notes, Princeton University Press and the University of Tokyo Press, Princeton, N.J., 1968.

Department of Mathematics, University of Oslo, Norway.

Permanent address: Department of Mathematics, University of Glasgow, Glasgow G12 8QW, SCOTland.

E-mail address: a.baker@maths.gla.ac.uk

$U R L:$ http://www.maths.gla.ac.uk/ ajb

E-mail address: h.s.e.gilmour@durham.ac.uk

E-mail address: p.reinhard@maths.gla.ac.uk 